\title{
New Approach of Automatic Modulation Classification based on in Phase-Quadrature Diagram Combined with Artificial Neural Network
}

\author{
Jean Baptiste Bi Gouho ${ }^{1}$, Désiré Melèdje ${ }^{2}$, Boko $\mathrm{Aka}^{3}$, Michel Babri ${ }^{4}$ \\ Laboratoire de Mathématique Informatique, Université Nangui Abrogoua, Abidjan, Côte d'Ivoire ${ }^{1,2,3}$ \\ Institut de Recherche sur les Energies Nouvelles, Université Nangui Abrogoua, Abidjan, Côte d'Ivoire ${ }^{3}$ \\ Institut National Polytechnique Félix Houphouët Boigny, Yamoussoukro, Côte d'Ivoire ${ }^{4}$
}

\begin{abstract}
Automatic Modulation Classification (AMC) with intelligent system is an attracting area of research due to the development of SDR (Software Defined Radio). This paper proposes a new algorithm based on a combination of k-means clustering and Artificial Neural Network (ANN). We use constellation diagram I-Q (In phase, Quadrature) as basic information. K-means algorithm is used to normalize data transmitted and pollute by the Additive White Gaussian Noise (AWGN), then the new diagram obtained is considered as an image and coded in pixel before entering in MLP (Multi-Layer Perceptron) Neural Network. Simulation results show an improvement of recognition rate under low SNR (Signal Noise Rate) compare to some results obtained in the literature.
\end{abstract}

Keywords-Modulations; Artificial Neural Network; clustering; machine learning

\section{INTRODUCTION}

Automatic Modulation Classification has an important research activity from the last decade $[1,2]$. That is due to his civilian and military applications. Principal goal of AMC is to classify different kind of modulations that has been intercepted. With the development of Artificial Neural Network (ANN) [3] and software Defined Radio [4], Liu et al [5] used fuzzy c-means combined with Artificial Neural Network for classify different type of modulations. The model proposed by Liu et al process certain parameters before entering in the neural network in the purpose of modulation recognition. Okhtay et al [6] proposed an algorithm of classification that use a combination of clustering algorithm kmeans and k-center using I-Q diagram of constellation for modulation classification. Zhechen et al used $\mathrm{K}$ Nearest Neighbor (KNN) combined with genetic algorithm [15] to achieve classification. $\mathrm{KNN}$ is one of famous learning algorithms which is categorized into learning and classification phases [14]. The principal problem is to classify modulated numerical signal, at the first step many papers $[5,6,15,16]$ used combination of 2 methods to solve the problem. We proposed a new approach based on I-Q diagram of constellation coded in pixel. At the first step we show that application of k-means algorithm alone in the purpose of normalizing the diagram polluted cannot be used at this point to recognize the modulation used. Then we combined the result obtained with neural network to help in classification but here I-Q diagram of constellation is considered as an image that will be used to feed the MLP neural network. In this paper, we propose an approach of modulation classification using k-means algorithm combined with artificial neural network. The work will be organized as follows:

The model used in this approach will be exposed in Section 2. Section 3 will present our approach of classification. Simulations results will be exposed in Section 4 and Section 5 is for future work and perspectives.

\section{REFERENCE MODEL}

We used as reference the model proposed by Liu et al [5] the model is presented in Fig. 1.

In this model modulated signal can be BPSK, QPSK, 8PSK, 4-QAM, 32-QAM, 64-QAM, etc.

When one of these modulations enumerated is used the signal is corrupted by Additive White Gaussian Noise (AWGN) which can affect the signal and cause an improbable demodulation. At the first step a clustering algorithm (Fuzzy $\mathrm{C}$-Means, K-means, K-center) is used in the purpose of normalizing data corrupted by noise. After that Artificial Neural Network (ANN) is used to recognize the type of modulation used.

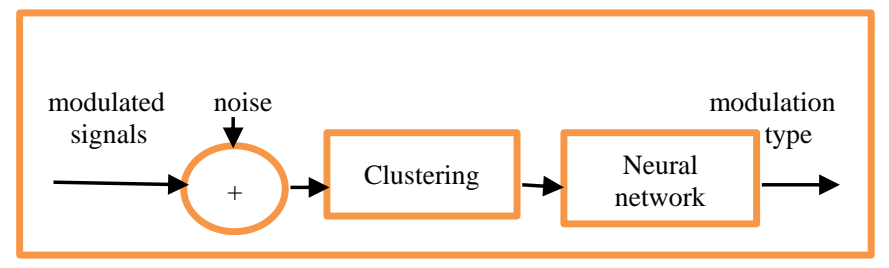

Fig. 1. System Model.

\section{PROPOSED Model of ClASSIFICATION}

In our model for classification, we suppose that Gaussian test for detecting single carrier or multiple carrier signal have been done [6]. In our case we are in face of single carrier signal. Clustering algorithm that we used is K-means.

\section{A. K-Means Algorithm}

Principle of k-means is to minimize the distortions that we call cost:

$$
\cos t=\min _{C}\left[\sum_{k=1}^{K} \sum_{x_{i} \in c_{k}}\left\|x_{i}-\mu_{K}\right\|^{2}\right]
$$


Where $x_{i}$ is a number of clusters $C_{k}$ and $\mu_{k}$ is the centroid of each cluster $C_{k}$.

$$
\mu_{K}=\frac{1}{N_{K}} \sum_{x_{i} \in K} x_{i}
$$

Fundamental purpose of clustering method is to assign observations into cluster. Mobasseri et al [13] show that receptor can use constellation of signal modulated to find the modulation used by emitter. To get the result he just consider the matrix of data $\mathrm{X}$ where $\mathrm{X}=\left[\begin{array}{l}\mathrm{x}_{1} \mathrm{x}_{2} . . \mathrm{x}_{\mathrm{j}} . . \mathrm{X}_{\mathrm{N}}\end{array}\right]^{\mathrm{T}}$. N represents an ensemble of points. $X_{j}=\left[\begin{array}{ll}x_{j i} & x_{j Q}\end{array}\right]^{T}$ where $x_{j i}$ is the component "in phase" and $\mathrm{x}_{\mathrm{jQ}}$ id the component in "quadrature".

When a signal modulated is for example 16 QAM with $\mathrm{N}$ $=10000$ and $\mathrm{S} / \mathrm{N}=4 \mathrm{~dB}$ we get the following result in Fig. 2 .

When a signal is transmitted and polluted by noise, we get the following result in Fig. 3.

After application of k-means clustering we have the result in Fig. 4.

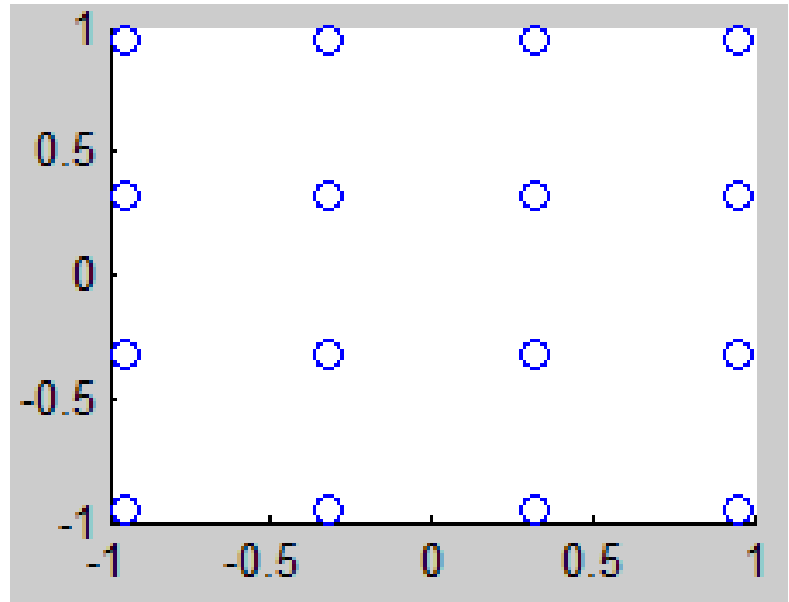

Fig. 2. Transmitted Signal.

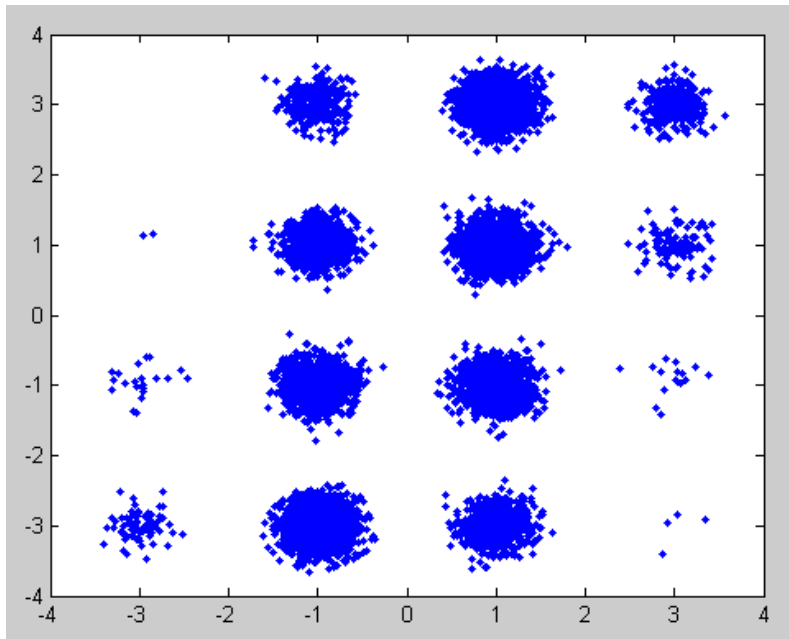

Fig. 3. Received Signal Polluted by the Noise.

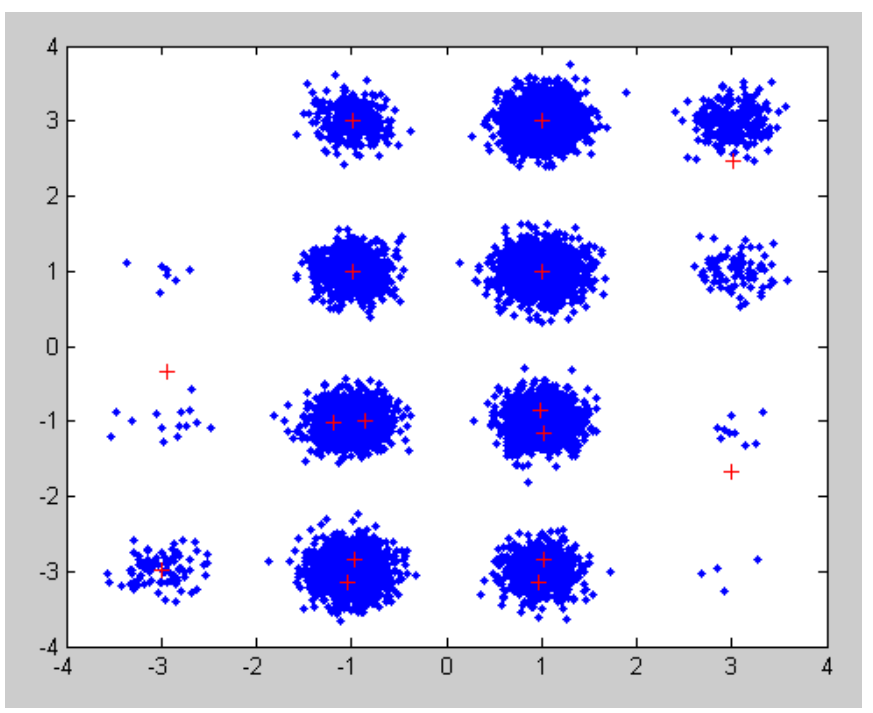

Fig. 4. Application of K-Means.

\section{B. Artificial Neural Network}

We use a Multilayer Perceptron (MLP) Neural Network [10] to solve our problem. In our case we have 7 classes corresponding to our 7 modulations chosen which are the most used in many papers $[5,6,8,11,12,15,16]$. As each modulation constellation can be set on a matrix $7 \times 7$, we set 49 input nodes. In addition, as we have 7 modulations to classify, the network contains 7 output nodes and the hidden layer has 98 nodes. Our MLP is presented in Fig. 5.

Activation function for neural network is "softmax function"

- Class1 $\Rightarrow$ [1000000] (16-QAM)

- Class $2 \Rightarrow$ [0100000] (4-QAM)

- Class3 $\rightarrow$ [0010000] (16-PSK)

- Class4 $\Rightarrow$ [0001000] (8-PSK)

- Class5 $\Rightarrow$ [0000100] (BPSK)

- Class6 $\rightarrow$ [0000010] (8-QAM)

- Class $\Rightarrow$ [0000001] (32-QAM)

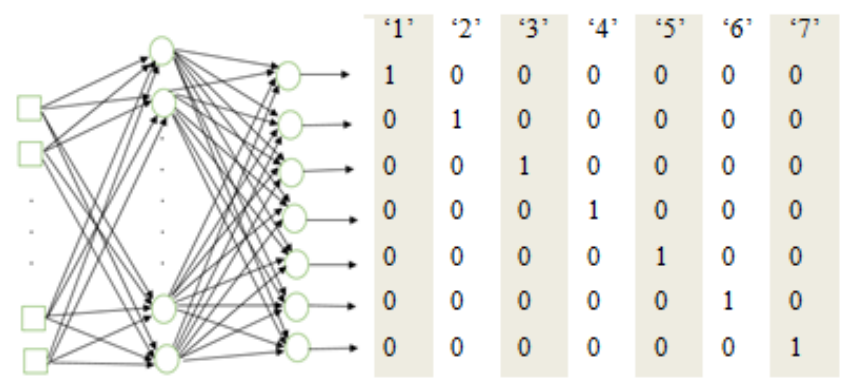

Fig. 5. Multi-Layer Perceptron.

$$
y_{i}=\varphi\left(v_{i}\right)=\frac{e^{v_{i}}}{\sum_{k=1}^{M} e^{v_{k}}}
$$




\section{Model for Modulation Classification}

Our model is a case of supervised learning where different modulations in use are known by receptor. I-Q diagram of constellation will be used as an image coded in pixel. For example, a constellation of a signal modulated in 16-QAM in our model called ANNG (Artificial Neural Network imaGe) will be coded as it is showed in Fig. 6.

Signal modulated in 16-QAM at $\mathrm{S} / \mathrm{N}=4 \mathrm{~dB}$ after applying $\mathrm{k}$-means algorithm and coded will be presented as it is on Fig. 7.

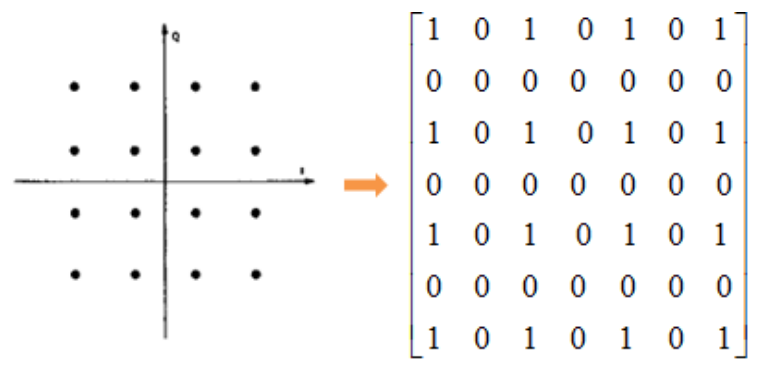

Fig. 6. 16-QAM Constellation Coded.

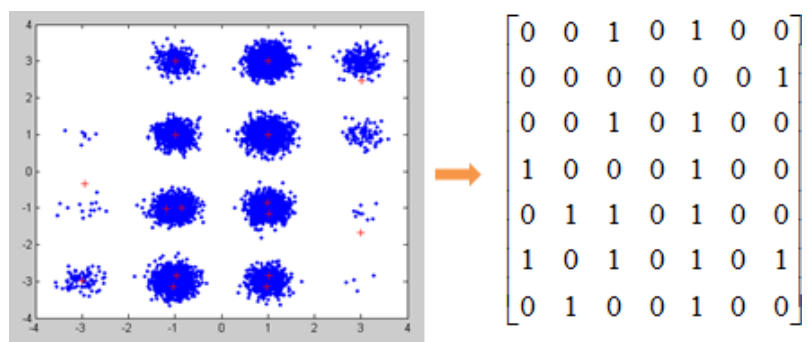

Fig. 7. 16-QAM Constellation Polluted by Noise Coded after Application of K-Means.

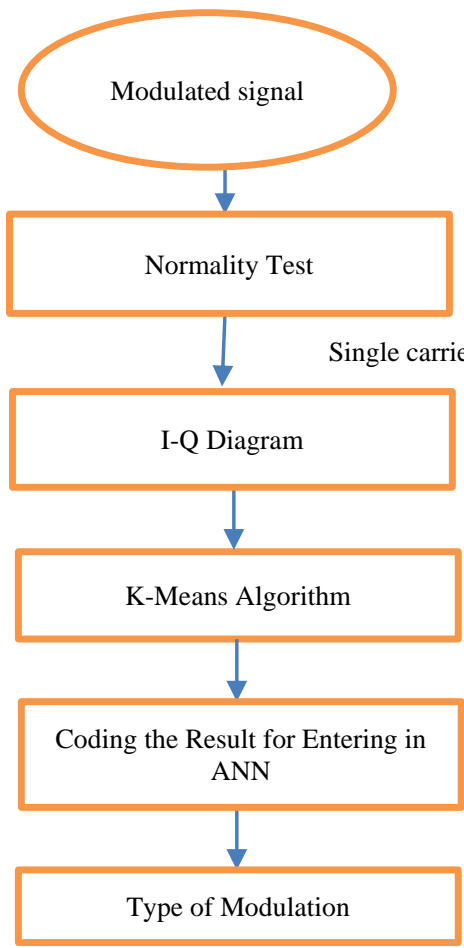

Fig. 8. Proposed Algorithm ANNG.
This result shows that for our model of classification constellation diagram I-Q can be considered as a matrix $7 \mathrm{x} 7$.

\section{Algorithm Diagram for Modulation Classification}

Proposed algorithm is presented in Fig. 8.

\section{RESULTS AND DISCUSSIONS}

In this part, we will show the results of our simulations. Table I present order of entrance in neural network.

Neural network will pass by a learning phase where epoch will take the value of 10,000 at the first stage and we will be increased to check for learning level of our model. We take the learning rate at $\alpha=0.9(0<\alpha \leq 1)$ because if that value is low neural network will converge slowly [7]. Our simulation has been done with these parameters:

- PC running Windows 10

- $8 \mathrm{~Gb}$ of RAM

- Processor Intel core i5 $7^{\text {th }}$ generation

- Programming language is MATLAB 2013a

In most of results found in different papers $[5,6,8,13,15,16]$ problem occurs when we face low value of $\mathrm{S} / \mathrm{N}$. That is why we used to test our model at $\mathrm{S} / \mathrm{N}=4 \mathrm{~dB}$. Fig. 9 presented an example of results.

Table II summarizes our simulation result.

This gives an average error rate for each epoch in Table III.

We know that an epoch corresponds to learning on all the data. The curve in Fig. 10 shows us that higher the number is better the accuracy is, which proves that our neural network behaves normally (convergence) with respect to the learning phase. But we note that from 4,000,000, the value of the number of iterations of our model has reached the critical point.

TABLE. I. MODULATIONS CHOSEN FOR CLASSIFICATION

\begin{tabular}{|l|l|}
\hline Order & Diagram I-Q pollute by noise \\
\hline 1 & 16-QAM \\
\hline 2 & 8-PSK \\
\hline 3 & 4-QAM \\
\hline 4 & BPSK \\
\hline 5 & 8-QAM \\
\hline 6 & 16-PSK \\
\hline 7 & 32-QAM \\
\hline
\end{tabular}

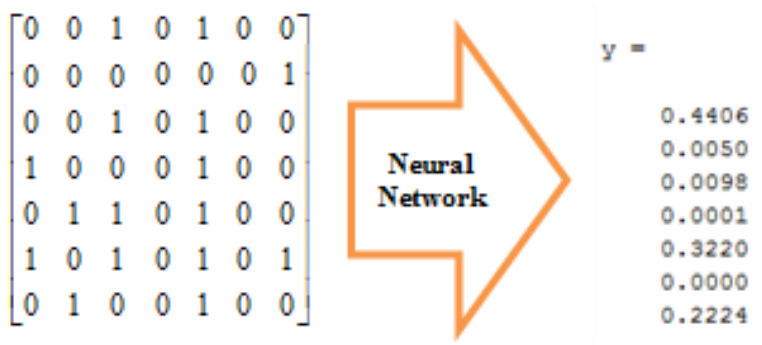

Fig. 9. An Example of Test Results. 


\section{convergence of neural network}

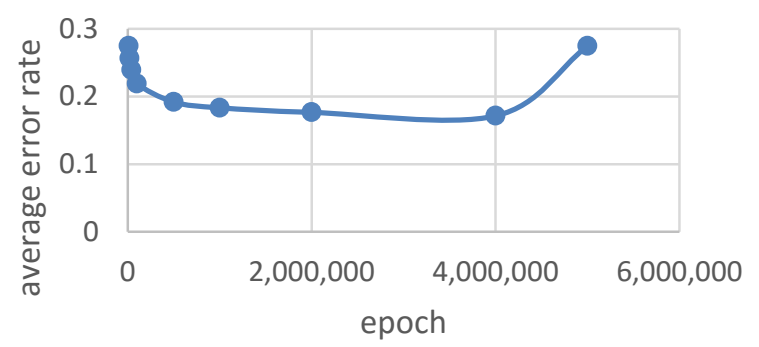

Fig. 10. Convergence of the Neural Network.

Tables II and III shows that our model gives good results on average percentage of total recognition of our seven modulations chosen is $82.85 \%$ at the cycle of iteration equal to $4,000,000$. Taking into account our chosen modulations, the weak recognition results are for 16 QAM and 16 PSK modulations. This is because: in our representation of the constellation of these modulations in the form of $7 \times 7$ matrix, there are many points more precisely 8 out of 16 points are placed at the same location on the constellation diagram presented in Fig. 11.

But it is on the basis of these constellations that our supervised learning happens with the neural network, which explains why the neural network sees practically in equal proportion:

- $45.66 \%$ for $16 \mathrm{QAM}$

- $53.45 \%$ for 16 PSK

TABLE. II. RESUlt OF THE NEURAL NETWORK FOR EACH MODULATION ACCORDING TO THE EPOCHS

\begin{tabular}{|l|l|l|l|l|l|}
\cline { 1 - 4 } Epoch & \multirow{2}{*}{$\mathbf{1 0 0 0 0}$} & $\mathbf{1 0 0 0 0 0}$ & $\mathbf{2 0 0 0 0 0 0}$ & $\mathbf{4 0 0 0 0 0 0}$ & $\mathbf{5 0 0 0 0 0 0}$ \\
\cline { 1 - 5 } Modulation & & & & & \\
\hline 16 QAM & 0,4406 & 0,497 & 0,4789 & 0,4566 & 0,4406 \\
\hline BPSK & 0,8124 & 0,8381 & 0,913 & 0,9291 & 0,8124 \\
\hline 8 PSK & 0,9914 & 0,9894 & 0,991 & 0,9917 & 0,9914 \\
\hline 8 QAM & 0,6541 & 0,83 & 0,9109 & 0,9193 & 0,6541 \\
\hline 4 QAM & 0,2333 & 0,35 & 0,5011 & 0,5345 & 0,2333 \\
\hline 32 QAM & 0,9506 & 0,9653 & 0,969 & 0,9687 & 0,9506 \\
\hline
\end{tabular}

TABLE. III. AVERAGE ERROR RATE OF RECOGNITION FOR EACH EPOCH

\begin{tabular}{|l|l|l|l|l|l|}
\hline epoch & $\mathbf{1 0 0 0 0}$ & $\mathbf{1 0 0 0 0 0}$ & $\mathbf{2 0 0 0 0 0 0}$ & $\mathbf{4 0 0 0 0 0 0}$ & $\mathbf{5 0 0 0 0 0 0}$ \\
\hline \multirow{6}{*}{} & 0,5594 & 0,503 & 0,5211 & 0,5434 & 0,5594 \\
\cline { 2 - 6 } & 0,1876 & 0,1619 & 0,087 & 0,0709 & 0,1876 \\
\cline { 2 - 6 } & 0,0086 & 0,0106 & 0,009 & 0,0083 & 0,0086 \\
\cline { 2 - 6 } & 0,3459 & 0,17 & 0,0891 & 0,0807 & 0,3459 \\
\cline { 2 - 6 } & 0,7667 & 0,65 & 0,4989 & 0,4655 & 0,7667 \\
\cline { 2 - 6 } & 0,0494 & 0,0347 & 0,031 & 0,0313 & 0,0494 \\
\cline { 2 - 6 } & 0,0054 & 0,0018 & 0,0003 & 0,0002 & 0,0054 \\
\hline Average & $\mathbf{0 , 2 7 4 7}$ & $\mathbf{0 , 2 1 8 8}$ & $\mathbf{0 , 1 7 6 6}$ & $\mathbf{0 , 1 7 1 4}$ & $\mathbf{0 , 2 7 4 7}$ \\
\hline
\end{tabular}
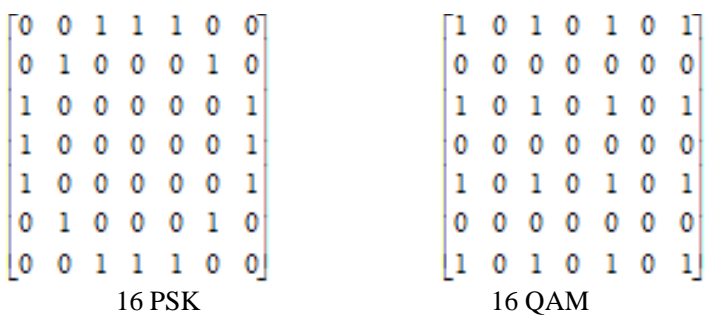

Fig. 11. Representation of the Constellations of 16 PSK and 16 QAM Modulations in Matrix Form.

Apart from these two modulations the results given are satisfactory. Table IV summarizes the results of recognition of the other modulations still at the cycle of iteration equal to $4,000,000$ which gives our best results.

\section{A. Comparison with other Results}

In the field of automatic modulation classification, the comparison directly with other results is difficult as was mentioned in $[13,16]$ this is because:

- each author uses certain parameters for their algorithms which are different from each other.

- there are no standard databases of modulations that each author must use in order to facilitate comparisons.

In case of comparing our results to some of the results presented in the literature, we choose four modulations (BPSK, 8 PSK, 4 QAM, 32 QAM) which are the modulations frequently used in many papers.

Table $\mathrm{V}$ presents the main results of some methods proposed in the literature.

TABLE. IV. RESULTS FOR FIVE MODULATIONS

\begin{tabular}{|l|l|}
\hline Modulations & Percentage of recognition \\
\hline BPSK & $92,91 \%$ \\
\hline 8 PSK & $99,17 \%$ \\
\hline 8 QAM & $91,93 \%$ \\
\hline 4 QAM & $96,87 \%$ \\
\hline 32 QAM & $99,98 \%$ \\
\hline average & $\mathbf{9 6 , 1 7} \%$ \\
\hline
\end{tabular}

TABLE. V. COMPARISON WITH OTHER METHODS

\begin{tabular}{|l|l|l|l|l|l|}
\hline Modulation & BPSK & 8 PSK & 4 QAM & $\begin{array}{l}\text { 32 } \\
\text { QAM }\end{array}$ & average \\
\hline $\begin{array}{l}\text { Muzzy Clustering } \\
\begin{array}{l}\text { Method (FCM) } \\
\text { of Liu et al }\end{array}\end{array}$ & $100 \%$ & $95.8 \%$ & $93.6 \%$ & $16 \%$ & $\mathbf{7 0 . 6} \%$ \\
\hline $\begin{array}{l}\text { Clustering } \\
\text { combined with } \\
\text { ANN of Liu et al }\end{array}$ & $100 \%$ & $100 \%$ & $100 \%$ & $73.6 \%$ & $\mathbf{9 1 . 2} \%$ \\
\hline $\begin{array}{l}\text { k-means and } \\
\text { k-center of } \\
\text { Ohktay et al }\end{array}$ & $100 \%$ & $87 \%$ & $96 \%$ & $95 \%$ & $\mathbf{9 4 . 5} \%$ \\
\hline $\begin{array}{l}\text { KNN } \\
\text { of Zhechen et al }\end{array}$ & $100 \%$ & 82.73 & $59.99 \%$ & $60 \%$ & $\mathbf{7 5 . 6 8 \%}$ \\
\hline $\begin{array}{l}\text { ANNG our } \\
\text { method }\end{array}$ & 92.91 & 99.17 & 96.87 & 99.98 & $\mathbf{9 7 . 2 3 \%}$ \\
\hline
\end{tabular}


As presented in Table $\mathrm{V}$, our method shows an improvement in term of recognizing different modulations used at low S/N particularly at $4 \mathrm{~dB}$.

\section{CONCLUSION}

In this paper, we presented a neural network-based modulation classification approach that we called ANNG (Artificial Neural Network imaGe). The originality of this approach is that it considers the I-Q diagram as an image that will be coded as a pixel. In the literature the input parameters of the neural network are calculated before entering in the neural network. Our new approach doesn't need to calculate certain value before entering in the neural network but just consider I-Q diagram as one block of image polluted by noise. At the first hand we use k-means clustering to normalize the diagram polluted by noise and after that use MLP to take a decision about the modulation used. Future work can be to reduce processor time consuming of our model [9] and improve percentage of recognition modulation. In this case we will try constructing a DNN (Deep Neural Network) of our model.

\section{REFERENCES}

[1] Zhu, Z., Nandi, A.K, "Automatic Modulation Classification : Principales, Algorithms and Applications ; John Wiley \& Sons 2015.

[2] O. A. Dobre., A. Abdi., Y. Bar-Ness., and W. Su., Survey of automatic modulation classification techniques: 362 classical approaches and new trends, IET Communications, vol. 1, no. 2, pp. 137-156, April 2007.

[3] Y. LeCun., Y. Bengio., and G. Hinton., Deep learning, Nature, vol. 521, no. 7553, pp. 436-444, May 2015.

[4] E. Grayver, Implementing Software Defined Radio; Springer Science+Business Media New York 2013.

[5] Liu, A., Zhu, Q., Automatic Modulation Classification based on the combinaison of clustering and neural network, the Journal of China Universities of Posts and Telecommunications, august 2011.
[6] Okhtay, A., Sven, G., I-Q diagram utilization in a novel modulation classification technique for cognitive radio applications, EURASIP Journal on Wireless Communications and Networking, 2013.

[7] P. Kim, Matlab Deep learning : with Machine Learning Neural Network and Artificial Intelligence, Apress, 2017.

[8] Guan Q. Y., Modulation Classification Based on Extensible Neural Networks, Hindawi Mathematical Problems in Engineering Volume 2017.

[9] Nelly, E., Anthony S. M., Magdy B. , Deep gated recurrent and convolutional network hybrid model for univariate time series classification, International Journal of Advanced Computer Science and Applications (IJACSA), Vol. 10, No 5, 2019.

[10] Asadur. R., Farzana. K., Kazem. H., Mohammad. K. A., Mohiuddin. Ahmad., Four-class motor imagery EEG signal classification using PCA, wavelet and two-stage neural network, International Journal of Advanced Computer Science and Applications (IJACSA), Vol. 10, No $5,2019$.

[11] Zhi, L. T., Si, M. L. and Li, J., Y. Implementation of Deep LearningBased Automatic Modulation Classifier on FPGA SDR Platform, MDPI/journal of electronics 2018.

[12] Jung, H. L., Jaekyum, K., Byeoungdo K., Dongweon, Y. and Jun, W. C. Robust Automatic Modulation Classification Technique for Fading Channels via Deep Neural Network MDPI/journal of entropy 2017.

[13] Bijan G. Mobasseri, Digital modulation classification using constellation shape, Signal Processing 80 (2000) p 251-277, Elsevier Science B.V.

[14] Jingwei, T., Abdul, R. A., Norhashimah, M. S. , Classification of hand movements based on discrete wavelet transform and enhanced feature extraction, International Journal of Advanced Computer Science and Applications (IJACSA), Vol. 10, No 6, 2019.

[15] Zhechen, Z., Aslam, M. W., Nandi, A.K, Automatic modulation classification using combination of genetic programming and KNN, IEEE transactions on wireless communications, 11 (8), 2742-2750, 2012.

[16] M.L.D Wong, A.K. Nandi, Automatic digital modulation recognition using artificial neural network and genetic algorithm, Signal Processing 84 (2004) p 351-365, Elsevier Science B.V. 